\title{
Major depression, 5HTTLPR genotype, suicide and antidepressant influences on thalamic volume
}

\author{
Keith A. Young, Willy L. Bonkale, Leigh A. Holcomb, Paul B. Hicks and Dwight C. German
}

\section{Background}

The 5HTTLPR genetic variant of the serotonin transporter gene (SERT or 5-HTT), which is comprised of a short (SERT-S) and a long (SERT-I) allele, is associated with major depressive disorder and post-traumatic brain disorder.

\section{Aims}

The present study sought to determine whether the total thalamus and major subregions are altered in size in major depressive disorder and in relation to the 5HTTLPR genotype.

\section{Method}

We investigated the influence of 5HTTLPR genotype, psychiatric diagnosis, suicide and other clinical factors on the volume of the entire post-mortem thalamus.

\section{Results}

Major depressive disorder, SERT-SS genotype and suicide emerged as independent factors contributing to an enlargement of the total thalamus. The majority of the volume enlargement associated with the SERT-SS genotype occurred in the pulvinar, whereas enlargement associated with major depressive disorder occurred in the limbic nuclei and in other regions of the thalamus. A history of antidepressant treatment was associated with reduced thalamic volume.

\section{Conclusions}

The 5HTTLPR genetic variation may affect behaviour and psychiatric conditions, in part, by altering the anatomy of the thalamus.

\section{Declaration of interest}

None. Funding detailed in Acknowledgements.
The serotonin transporter (SERT or 5-HTT) regulates brain serotonin (5-HT) neurotransmission by removing neurotransmitter from the extracellular space. Genetic variation in the promoter region of the SERT gene (5HTTLPR) is common, and the short (SERT-s) and long (SERT-1) alleles of 5HTTLPR are emerging as important mediators of emotional temperament and predisposition to mental illness. Inheritance of SERT-s has been associated with an increased incidence of both major depressive disorder and post-traumatic stress disorder, but not other psychiatric conditions such as schizophrenia and bipolar disorder. ${ }^{1,2,3}$ The finding that lymphoblastoid cell lines from individuals homozygous for the SERT-s allele (SERT-ss) have approximately half the 5-HT uptake capacity of cells from SERT-l carriers has led to the expectation that SERT levels would be found to be reduced in individuals with a SERT-ss genotype. ${ }^{4}$ However, data obtained to date suggest that SERT expression in the human central nervous system is largely independent of 5HTTLPR genotype, raising the question of how this genetic variant influences behaviour and mental illness. ${ }^{5}$ Recently, evidence for a robust 5HTTLPR influence on brain anatomy has emerged. The SERT-s allele has been associated with a $25 \%$ reduction in volume of the anterior cingulate cortex and amygdala, and a $20 \%$ increase in neuron number and volume in the pulvinar nucleus of the thalamus. ${ }^{6,7}$ The pulvinar, cingulate and amygdala are interconnected nodes in the limbic system involved in mediating such functions as attention to the threat content of the environment and emotional response to pain. ${ }^{8}$ Association of enlargement of the pulvinar nucleus of the thalamus with SERT-ss genotype is a particularly interesting observation, since this enlargement could potentially contribute to a 'gain in function' resulting in accentuated emotional responses to environmental stimuli, with the additional input to the limbic system contributing to altered limbic functioning and anatomical deficits present in the amygdala, frontal cortex and cingulate lobe. In the present study, we extended the investigation of the role of the 5HTTLPR genotype and other clinical factors on the volume of the entire post-mortem thalamus. We sought to determine whether the enlargement of the pulvinar in individuals with SERT-ss genotype is specific for the pulvinar region, or whether the entire thalamus is enlarged in these individuals.

\section{Method}

A total of 54 specimens from the Stanley Foundation Brain Collection with a complete series of thalamic sections matched for age, gender and post-mortem interval were utilised. ${ }^{9}$ Controls were never diagnosed with psychiatric illness. Individuals with a psychiatric illness were diagnosed with major depressive disorder, bipolar disorder or schizophrenia. About half died by suicide. Individuals from each of the three psychiatric diagnoses, and from suicide (12/17) and non-suicide (11/22) categories, had been treated with antidepressants. Information on age, post-mortem interval, time in formalin, gender and hemisphere sampled is provided in Table 1.

The entire thalamus from the formalin-fixed hemisphere was serially sectioned at $60 \mu \mathrm{m}$ thickness in the coronal plane, mounted on glass slides and stained for Nissl with cresyl violet. ${ }^{10}$ Thalamic borders were outlined in Nissl-stained sections using a microscopic imaging system. The lateral thalamic boundary was defined as the border of the reticular nucleus, and the ventrolateral border was defined by the medial geniculate nucleus. The lateral geniculate nucleus was not included in the total thalamic volume assessment. Cavalieri procedure (StereoInvestigator PC software, version 6; MicroBrightField Inc, Williston, Vermont, USA) was used to estimate thalamic volumes. Results are reported as volume corrected for post-sectioning shrinkage in the $z$-axis.

A sample of DNA was extracted from frozen cerebellar tissue using the QIAAmp DNA mini-kit, Qiagen, and 5HTTLPR genotype was determined in duplicate assays using polymerase chain reaction. ${ }^{11}$ Oligonucleotide primers flanking 5HTTLPR and corresponding to the nucleotide positions 1416 to 1397 (STPR5, 
Table 1 specimen characteristics

\begin{tabular}{|c|c|c|c|c|}
\hline & Control & Major depressive disorder & Bipolar disorder & Schizophrenia \\
\hline$n$ & 15 & 14 & 13 & 12 \\
\hline Age, years: mean (s.d.) & $48.1(10.7)$ & 45.2 (8.1) & $43.7(12.0)$ & $44.3(13.5)$ \\
\hline Post-mortem interval, h: mean (s.d.) & $23.7 \quad(9.9)$ & $28.0(10.8)$ & $31.8(17.0)$ & $34.1(16.3)$ \\
\hline Brain weight, g: mean (s.d.) & $1501(164)$ & $1469(145)$ & $1453(156)$ & $1467(111)$ \\
\hline Time in formalin, months: mean (s.d.) & 4.4 (3.4) & $8.0 \quad(6.6)$ & $9.3 \quad(3.7)$ & $12.3(9.1)$ \\
\hline Left hemisphere sampled, \% & 46 & 36 & 54 & 42 \\
\hline Female, \% & 40 & 43 & 38 & 33 \\
\hline
\end{tabular}

5'-GGCGTTGCCGCTCTGAATGC) and 910 to 888 (STPR3, 5'GAGGGACTGAGCTGGACAACCAC) were used to generate 484- and 528-base-pair fragments. The assay consisted of a modified touchdown polymerase chain reaction protocol with an antibody-mediated hot start. The polymerase chain reaction products were visualised by running on a $1.8 \%$ agarose gel with ethidium bromide.

Owing to the many uncontrolled clinical and post-mortem variables present in the cohort, our primary analysis was performed with analysis of covariance (ANCOVA: JMP Macintosh software, version 5.01a) of the whole cohort (all individuals $n=54)$. The ANCOVA was repeated for individuals with psychiatric illness alone $(n=39)$ so that we could analyse the effects of additional variables present only in these individuals. Covariates in the complete ANCOVA were diagnosis, age, gender, hemisphere, time in formalin and post-mortem interval, with suicide and treatment with antidepressants added as additional cofactors in the ANCOVA subgroup of individuals with psychiatric illness. A truncated genotype categorisation was used for the primary analysis (SERT-ss $v$. SERT-sl/ll) in order to maintain statistical power. After these primary analyses were complete, we performed exploratory testing such as reanalysing the data with a full genotype categorisation (SERT-ss $v$. SERT-sl $v$. SERT-ll).

\section{Results}

Overall, 14 people possessed the SERT-ss genotype (bipolar disorder $2 / 13$, major depressive disorder $5 / 14$, schizophrenia $5 / 12$, controls 2/15), and 16 possessed the SERT-ll genotype (bipolar disorder 5/13, major depressive disorder 3/14, schizophrenia 2/12, controls 6/15). People with a SERT-ss genotype were not significantly different with respect to age, gender, suicide, diagnosis, substance or alcohol misuse from people with a SERT-sl/ll genotype $(P>0.05)$.

\section{Total thalamic volume}

For the analysis of the 54 samples from all four diagnostic categories, covariate effects were observed for SERT genotype (ss $>$ sl/ll), diagnosis (major depressive disorder $>$ others), gender (male $>$ female), age (4.6\% reduction per decade), but not hemisphere, time in formalin or post-mortem interval (see Table 2 and online Fig. DS1). Inspection of the ANCOVA least squared means indicates a significant $11 \%$ thalamic enlargement between individuals with SERT-ss genotype $v$. SERT sl/ll genotype, and a significant $12 \%$ enlargement in people with major depressive disorder compared with controls. The subgroup ANCOVA limited to people with psychiatric illness revealed similar covariate effects for SERT genotype, diagnosis, gender and age (Table 2). In addition, we observed significant covariate effects for time in formalin ( $0.3 \%$ reduction per month), hemisphere (right $>$ left), suicide (suicide $>$ non-suicide) and antidepressant medication (non-treatment $>$ treatment). Suicide was associated with an $8 \%$ enlargement of the thalamus, whereas antidepressant treatment was associated with an $18 \%$ lower total thalamic volume. The antidepressant effect was most evident in the schizophrenia group, which was relatively well-balanced for positive and negative histories of antidepressant usage. The nine covariate factors in the second ANCOVA produced a highly informative model predicting total thalamus volume $\left(r^{2}=0.793, P<0.0001\right)$. As a post hoc validation of the subgroup ANCOVA findings, we performed forward stepwise multiple regression for individuals with psychiatric illness alone. All of the above significant covariates, except for time in formalin and hemisphere, were identified in the stepwise regression model as significant and independent predictors of total thalamic volume. Also, we performed an exploratory ANCOVA using thalamic volume normalised for whole brain weight. The SERT-ss genotype and major depressive disorder diagnosis effects remained significant in this analysis, supporting a selective effect of these variables on thalamic volume as compared with the brain as a whole. To clarify SERT allele effects, we removed post-mortem interval (a non-significant factor in all models) and substituted the two-way with a three-way genotype categorisation (SERT-ss $v$. SERT-sl $v$. SERT-1l). In this exploratory ANCOVA, only individuals with SERT-ss genotype had an enlarged thalamus compared with individuals with either SERT-sl or SERT-ll genotypes; individuals with SERT-sl and SERT-ll genotypes were not significantly different from each other.

\section{Regional effects associated with SERT-sS and major depressive disorder}

We had previously determined volumes of mediodorsal, anteroventral/anteromedial and pulvinar nuclei in the samples used in the current study (Fig. 1). ${ }^{7,13}$ In the previous studies, we observed that major depressive disorder was associated with significant enlargement of the limbic (mediodorsal and anteroventral/ anteromedial) thalamus, and that SERT-ss genotype was primarily associated with enlargement of the pulvinar nuclei. The present study indicates a mean total thalamic volume enlargement of $654 \mathrm{~mm}^{3}$ associated with major depressive disorder, and of $444 \mathrm{~mm}^{3}$ associated with the SERT-ss genotype (Fig. 1). Our previously published data indicate that in the limbic thalamus there was a mean $167 \mathrm{~mm}^{3}$ volume enlargement associated with major depressive disorder, and in the pulvinar there was a mean $256 \mathrm{~mm}^{3}$ volume enlargement associated with the SERT-ss genotype. Therefore, we conclude that $26 \%\left(167 / 654 \mathrm{~mm}^{3}\right)$ of the total enlargement associated with major depressive disorder occurs in the limbic thalamus, and that $57 \%\left(256 / 444 \mathrm{~mm}^{3}\right)$ of the total enlargement associated with SERT-ss genotype occurs in the pulvinar. Figure 1 illustrates the localisation of SERT-ss and major depressive disorder enlargement effects in limbic and pulvinar subregions of the thalamus and, by subtraction, the enlargement that can be allocated to the remaining nuclei of the 


\begin{tabular}{|c|c|c|c|c|}
\hline \multirow[b]{2}{*}{ Covariate } & \multicolumn{2}{|c|}{ All individuals $(n=54)$} & \multicolumn{2}{|c|}{ Individuals with psychiatric illness $(n=39)$} \\
\hline & F ratio & $P$ & $F$ ratio & $P$ \\
\hline SERT-SS & 4.63 & 0.037 & 5.78 & 0.023 \\
\hline Diagnosis & 2.79 & 0.049 & 15.07 & 0.001 \\
\hline Age & 14.31 & 0.001 & 11.1 & 0.003 \\
\hline Gender & 4.09 & 0.049 & 19.9 & 0.001 \\
\hline Hemisphere & 0.28 & NS & 5.1 & 0.033 \\
\hline Post-mortem interval & 0.14 & NS & 2.1 & NS \\
\hline Time in formalin & 0.61 & NS & 5.0 & 0.033 \\
\hline Antidepressants ${ }^{a}$ & - & - & 41.6 & 0.001 \\
\hline Suicide $^{a}$ & - & - & 7.69 & 0.009 \\
\hline
\end{tabular}

thalamus. The data indicate that the majority of enlargement associated with SERT-ss occurs in the pulvinar, whereas a relatively large portion of that associated with major depressive disorder occurs outside of the limbic and pulvinar thalamic regions.

\section{Discussion}

The present data indicate that both the SERT-ss genotype and major depressive disorder influence the total volume of the thalamus. The thalamus is enlarged by approximately $11 \%$ in people carrying the 5HTTLPR SERT-ss genotype. A similar magnitude enlargement of the thalamus, independent from the 5HTTLPR effect, was observed in people with major depressive disorder compared with controls. Thalamic enlargement is not likely to be related to an overall larger brain in people with SERT-ss genotype and major depressive disorder because the thalamic enlargement effect remained significant after normalising for whole brain weight. Although we did not measure total thalamic neuron number in the present study, we have previously observed that both volume and neuron number are increased in the pulvinar in people with SERT-ss genotype and in the limbic thalamus of people with major depressive disorder., ${ }^{7,13}$

\section{Thalamic volume and SERT-SS}

The present data suggest that the SERT-ss thalamic enlargement is particularly robust in the pulvinar, since $57 \%$ of the total thalamic enlargement occurs in this nucleus (which occupies about $30 \%$ of the thalamus). We also found preliminary evidence that SERT-ss enlargement is present in the limbic thalamus (mediodorsal and anteroventral/anteromedial), where $26 \%$ of the total thalamic enlargement occurred. Combining these results, the great majority $(83 \%)$ of SERT-ss thalamic enlargement occurs in these two regions, which together comprise about a half of the entire thalamus. Both the pulvinar and limbic thalamus contain a dense plexus of SERT-containing serotonin fibres, with the pulvinar in particular being a site of very dense 5-HT innervation. It is striking that the pulvinar and midline thalamic regions, which contain a very dense plexus of SERT-containing fibres, are sites of major anatomic changes associated with inheritance of the SERT-ss genetic variation. ${ }^{14}$ The high density of SERT in these regions may provide a substrate for 5HTTLPR-associated alterations in 5-HT neurotransmission to affect both thalamic anatomy and function. In addition to the high levels of SERT present in the mature thalamus, SERT is also critically involved in shaping the anatomy of both the thalamus and cortex during development. For instance, in SERT knockout mice, the complex patterning of thalamocortical connectivity is altered, and there is a reduction in programmed cell death in the thalamus. ${ }^{15,16}$ Furthermore, during the period when thalamic fibres first reach the cortex, some glutamatergic thalamocortical neurons transiently express SERT on their axons. ${ }^{17}$ Further study is needed to investigate how 5HTTLPR genetic variation influences the development of the thalamus.

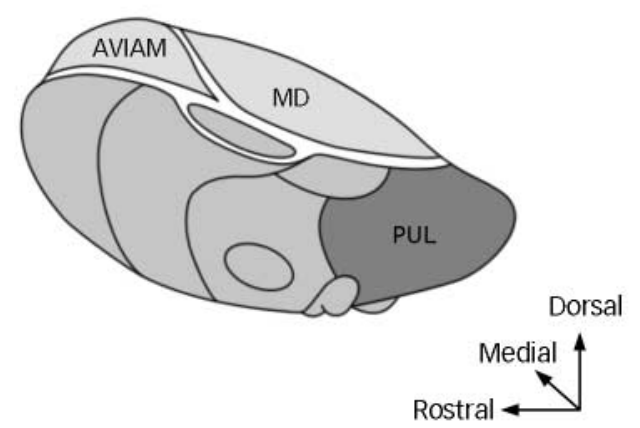

(a)

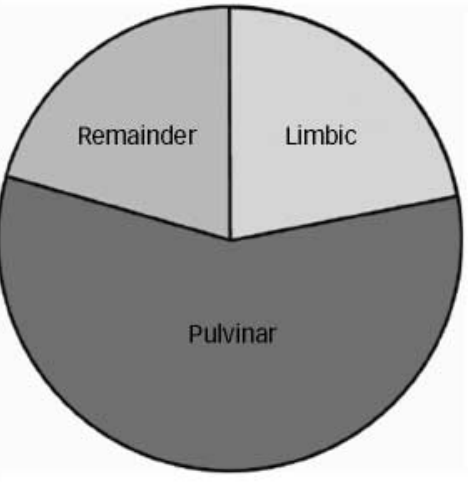

(b)

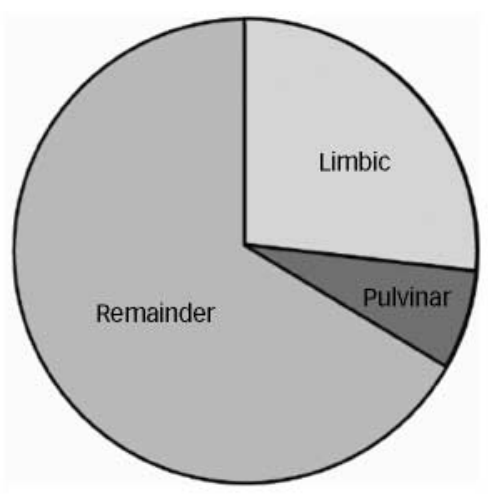

(c)

Fig. 1 Illustration of the thalamus.

(a) The location of the pulvinar (PUL) and limbic (mediodorsal (MD) and anteroventral/anteromedial (AV/AM)) regions of the thalamus. The remainder of the thalamus is comprised of a variety of nuclei. Pie charts: area enlarged in the SERT-ss genotype group (b) and the major depressive disorder group (c). The majority (57\%) of the total thalamic enlargement related to the SERT-SS genotype occurs in the pulvinar (b), whereas that associated with major depressive disorder occurs primarily outside of the pulvinar and limbic thalamus (c). Adapted from Carpenter \& Sutin. ${ }^{12}$ 
Anatomical changes in the thalamus associated with 5HTTLPR may contribute to genetic susceptibility to major depressive disorder, post-traumatic stress disorder and other conditions (e.g. suicide and anxiety). ${ }^{18}$ Functionally, the pulvinar processes information related to environmental threat, such as facially expressed fear, and relays this information to important nodes in the limbic system. ${ }^{8}$ Both the pulvinar and the limbic thalamus are intimately connected to the anterior cingulate cortex, a brain area involved in processing internally generated thoughts, emotional responses to pain and attention to negative consequences. In major depressive disorder, there is evidence that elevated levels of introspection, rumination (brooding) and attention to negative facial expressions are very common, and display trait rather than state variability. ${ }^{19}$ Given the intimate connections of the pulvinar and limbic thalamus to the limbic system, thalamic enlargement may contribute to behavioural problems in major depressive disorder and other mood disorders by facilitating activity of the anterior cingulate and other limbic centres. Interestingly, Greicius et al have shown that resting-state functional connectivity between the medial thalamus and the anterior cingulate is accentuated in major depressive disorder, with the most affected areas being found in the medial thalamus and pulvinar. ${ }^{20}$ Our data suggest that thalamic enlargement represents a possible explanation for this accentuated functional connectivity. Furthermore, because of the close anatomical connections between the thalamus and the limbic system, thalamic anatomical changes in people with SERT-ss genotype may directly contribute to 5HTTLPR-associated reductions in grey matter volume in the amygdala and cingulate cortex. ${ }^{6}$ These anatomical substrates may be future targets for surgical interventions. It has been demonstrated that deep brain stimulation of the white matter tracts connecting the thalamus with the anterior cingulate alleviates symptoms of severe depression. ${ }^{21}$ It will be interesting to determine whether the efficacy of deep brain stimulation is related to the interruption of excessive thalamic input to the cingulate cortex. Thus, although it is clear that 5HTTLPR genetic variation could have an effect on 5-HT neurotransmission and behaviour by altering SERT kinetics and affecting 5-HT levels in the mature brain, the present data support an alternative hypothesis implicating 5HTTLPR-related alterations in brain anatomy as being important factors in mediating major depressive disorder and other affective states. ${ }^{4}$

\section{Thalamic volume and psychiatric symptoms}

In addition to SERT-ss effects on the volume of the thalamus, we observed that people diagnosed with major depressive disorder have $12 \%$ larger thalami compared with controls. Previously, we reported that limbic nuclei, but not the pulvinar, were enlarged in people with major depressive disorder. ${ }^{7,13}$ However, since the previously observed limbic enlargement (mediodorsal and anteroventral/anteromedial) represents only $26 \%$ of the total thalamic enlargement, there must be a substantial enlargement in other thalamic regions in major depressive disorder. Possibilities for the site of this enlargement include nuclei of the ventral tier (ventroanterior, ventrolateral) and a variety of smaller nuclei (reticular, centromedian, laterodorsal, medial geniculate).

The present data suggest that death by suicide is associated with thalamic enlargement in people with psychiatric illness. Notice in online Fig. DS1 that many of the individuals that died by suicide (marked in red) had large thalami. Although other subcortical structures such as the adrenal glands and pituitary are enlarged in people likely to die by suicide, the present data represent the first evidence for enlargement of the thalamus in suicide. $^{22,23}$ Like major depressive disorder, suicide has been linked to developmental stress, including physical and sexual abuse, which have profound influences on brain serotonin. ${ }^{24,25}$ As described above, it may be possible that developmental stress and trauma exacerbate thalamic enlargement by potentiating 5HTTLPR effects on serotonergic neurotransmission during a critical developmental period. If that is the case, then thalamic enlargement may be an example of an anatomical substrate affected by a serotonergic gene $\times$ environment interaction. ${ }^{26}$

\section{Thalamic volume and antidepressants}

Many antidepressant drugs block reuptake of 5-HT by interacting directly with SERT. In the present study, a history of antidepressant use in people with psychiatric illness was associated with a smaller thalamic volume. This observation contrasts with antidepressant effects observed in the hippocampus, where the medication is associated with volume enlargement. ${ }^{27}$ The presence of smaller thalami in people with psychiatric illness with a history of treatment with antidepressants suggests either that thalamic tissue mass shrinks when individuals are administered antidepressants, or that individuals with larger thalami are less likely to be given antidepressants. Although it is tempting to conclude that antidepressant treatment interacts with the serotonergic system to reverse thalamic enlargement, further study is needed to determine whether this is indeed the case, or whether individuals that do not receive antidepressants comprise a unique group of people possessing relatively large thalami.

\section{Further research}

The present data indicate that there was a twofold variation in thalamic volume among the people investigated in this study. Multiple factors, including post-mortem, clinical and genetic factors, contributed to this variation. It is notable that the ANCOVA model produced by controlling for nine cofactors captured a substantial portion of this variation $\left(r^{2}=0.79\right)$. The success of the model sets limits on improvements that could be made by inclusion of additional genetic variants or clinical factors. Thus, careful attention to clinical factors such as SERT genetic background, suicidality and antidepressant treatment, not normally controlled for in structural brain studies, may provide a means to decrease variability and improve power in post-mortem and structural MRI studies.

In summary, the present data indicate that a diagnosis of major depressive disorder, SERT-ss genotype and suicide are associated with thalamic hypertrophy, whereas people with a history of treatment with antidepressants have smaller thalami. Individuals with enlarged thalami may have an anatomical vulnerability to stress, owing to alterations in thalamocortical circuit function. The present findings support the broad hypothesis that a 5-HT-linked brain structural phenotype, characterised in part by thalamic enlargement, may predispose individuals to symptoms of depression and to related behaviours such as suicide.

Keith A. Young, PhD, Willy L. Bonkale, PhD, Leigh A. Holcomb, PhD, Paul B. Hicks, MD, PhD, Neuropsychiatry Research Program, Central Texas Veterans Health Care System and Texas A\&M Health Science Center Department of Psychiatry and Behavioral Science, Temple, Texas; Dwight C. German, PhD, Department of Psychiatry, University of Texas Southwestern Medical Center, Dallas, Texas, USA

Correspondence: Keith A. Young, Neuropsychiatry Research Program, Central Texas Veterans Health Care System and Texas A\&M Health Science Center Department of Psychiatry and Behavioral Science, 1901 S. 1st Street, Temple, TX 76504, USA. Email: kayoung@medicine.tamhsc.edu

(First received 16 Apr 2007, final revision 27 Aug 2007, accepted 1 Oct 2007) 


\section{Acknowledgements}

We thank the Stanley Foundation Neuropathology Consortium (M. Knable, F. Torrey, M Webster and R. Yolken) for providing tissue used in this study. The technical assistance of Samantha Sachsenmaier and Umar Yazdani, and the editoral expertise of Laura Miller are greatly appreciated. The National Institute of Mental Health (MH59145), the Veteran Administration, the Zigenbein Memorial Fund, and the Theodore and Vada Stanley Foundation financially supported this work.

\section{References}

1 Lin PY, Tsai G. Association between serotonin transporter gene promoter polymorphism and suicide: results of a meta-analysis. Biol Psych 2004; 55: 1023-30.

2 Lee HJ, Lee MS, Kang RH, Kim H, Kim SD, Kee BS, Kim YH, Kim YK, Kim JB Yeon BK. Influence of the serotonin transporter promoter gene polymorphism on susceptibility to posttraumatic stress disorder. Depress Anxiety 2005; 21: 135-9.

3 Ikeda M, Iwata N, Suzuki T, Kitajima T, Yamanouchi Y, Kinoshita Y, Ozaki NJ. No association of serotonin transporter gene (SLC6A4) with schizophrenia and bipolar disorder in Japanese patients: association analysis based on linkage disequilibrium. J Neural Trans 2006; 113: 899-905.

4 Lesch KP, Bengel D, Heils A, Sabol SZ, Greenberg BD, Petri S, Benjamin J, Muller $\mathrm{CR}$, Hamer DH, Murphy DL. Association of anxiety-related traits with a polymorphism in the serotonin transporter gene regulatory region. Science 1996; 274: 1527-30.

5 Parsey RV, Hastings RS, Oquendo MA, Hu X, Goldman D, Huang YY, Simpson $\mathrm{N}$, Arcement J, Huang Y, Ogden RT, Van Heertum RL, Arango V, Mann JJ. Effect of a triallelic functional polymorphism of the serotonin-transporterlinked promoter region on expression of serotonin transporter in the human brain. Am J Psych 2006; 163: 8-11.

6 Pezawas L, Meyer-Lindenberg A, Drabant EM, Verchinski BA, Munoz KE, Kolachana BS, Egan MF, Mattay VS, Hariri AR, Weinberger DR. 5-HTTLPR polymorphism impacts human cingulate-amygdala interactions: a genetic susceptibility mechanism for depression. Nat Neurosci 2005; 8: 828-34.

7 Young KA, Holcomb LA, Bonkale WL, Hicks PB, Yazdani U, German DC. 5HTTLPR polymorphism and enlargement of the pulvinar: Unlocking the backdoor to the limbic system. Biol Psych 2007; 61: 813-18.

8 Skuse D. Genetic influences on the neural basis of social cognition. Philos Trans R Soc Lond B: Biol Sci 2006; 1476: 2129-41.

9 Torrey EF, Webster M, Knable M, Johnston N, Yolken RH. The Stanley Foundation Brain Collection and Neuropathology Consortium. Schizophr Res 2000; 44: 151-5

10 Prophet EB, Mills B, Arrington JB, Sobin LH. Armed Forces Institute of Pathology: Laboratory Methods in Histotechnology. American Registry of Pathology, 1992

11 Heils A, Mossner R, Lesch KP. The human serotonin transporter gene polymorphism: basic research and clinical implications. J Neural Trans 1997 104: $1005-14$

12 Carpenter MB, Sutin J. Human Neuroanatomy (8th edn). Williams \& Wilkins, 1983.
13 Young KA, Holcomb LA, Yazdani U, Hicks PB, German DC. Elevated neuron number in the limbic thalamus in major depression. Am J Psych 2004; 161 1270-7.

14 Jarkas N, McConathy J, Votaw JR, Voll RJ, Malveaux E, Camp VM, Williams L, Goodman RR, Kilts CD, Goodman MM. Synthesis and characterization of EADAM: a selective radioligand for mapping the brain serotonin transporters by positron emission tomography. NuCl Med Biol 2005; 32: 75-86.

15 Persico AM, Mengual E, Moessner R, Hall FS, Revay RS, Sora I, Arellano J, DeFelipe J, Gimenez-Amaya JM, Conciatori M, Marino R, Baldi A, Cabib S, Pascucci T, Uhl GR, Murphy DL, Lesch KP, Keller F. Barrel pattern formation requires serotonin uptake by thalamocortical afferents, and not vesicular monoamine release. J Neurosci 2001; 21: 6862-73.

16 Persico AM, Baldi A, Dell'Acqua ML, Moessner R, Murphy DL, Lesch KP, Keller $F$. Reduced programmed cell death in brains of serotonin transporter knockout mice. Neuroreport 2003; 14: 341-4.

17 Verney C, Lebrand C, Gaspar P. Changing distribution of monoaminergic markers in the developing human cerebral cortex with special emphasis on the serotonin transporter. Anat Rec 2002; 267: 87-93.

18 Serretti A, Calati R, Mandelli L, De Ronchi D. Serotonin transporter gene variants and behavior: a comprehensive review. Curr Drug Targets 2006; 7 1659-69.

19 Joormann J, Dkane M, Gotlib IH. Adaptive and maladaptive components of rumination? Diagnostic specificity and relation to depressive biases. Behav Ther 2006; 37: 269-80.

20 Greicius MD, Flores BH, Menon V, Glover GH, Solvason HB, Kenna H, Reiss AL, Schatzberg AF. Resting-state functional connectivity in major depression: abnormally increased contributions from subgenual cingulate cortex and thalamus. Biol Psych 2007; 62: 429-37.

21 Mayberg HS, Lozano AM, Voon V, McNeely HE, Seminowicz D, Hamani C, Schwalb JM, Kennedy SH. Deep brain stimulation for treatment-resistant depression. Neuron 2005; 45: 651-60.

22 Thomas LA, Debilis MD. Pituitary volumes in pediatric maltreatment-related posttraumatic stress disorder. Biol Psych 2004; 55: 752-8.

23 Dumser T, Barocka A, Schubert E. Weight of adrenal glands may be increased in persons who commit suicide. Am J Forensic Med Path 1998; 19 $72-6$

24 Mann JJ, Waternaux C, Haas GL, Malone KM. Toward a clinical model of suicidal behavior in psychiatric patients. Am J Psych 1999; 156: 181-9.

25 Bennett AJ, Lesch KP, Heils A, Long JC, Lorenz JG, Shoaf SE, Champoux M, Suomi SJ, Linnoila MV, Higley JD. Early experience and serotonin transporter gene variation interact to influence primate CNS function. Mol Psych 2002; 7: $118-22$.

26 Caspi A, Sugden K, Moffitt TE, Taylor A, Craig IW, Harrington H, McClay J, Mill J, Martin J, Braithwaite A, Poulton R. Influence of life stress on depression: moderation by a polymorphism in the 5-HTT gene. Science 2003; 301: 386-9.

27 Vermetten E, Vythilingam M, Southwick SM, Charney DS, Bremner JD. Longterm treatment with paroxetine increases verbal declarative memory and hippocampal volume in posttraumatic stress disorder. Biol Psych 2003; 54 693-702.

\section{Psychosis - the price Homo sapiens pays for language

Age- and sex-dependent uniformity across populations of the psychotic continuum reflects ontogeny of the cerebral torque. This sapiensspecific evolutionary innovation identified four compartments of association cortex as the language circuit: perceived speech $>$ meaning $>$ thought > speech production. Symptoms (due to directional asynchronies of callosal myelination) represent 'leakages' - negative anterior, positive posterior, linguistic to left, affective to right. The speciation event occurred 160KYA in the Y copy (PCDHY) of the ProtocadherinXY homologous gene pair with subsequent sequence change in PCDHX. Thus, language, H. sapiens \& predisposition to psychosis resulted from a single chance event in a male, selected by females. 